\title{
合成压力对 $\mathrm{FeOOH}$ 纳米固体结构 的影响研究 ${ }^{*}$
}

隋 郁 (1) 许大鹏 (1) 苏文辉 ${ }^{(1)}$ (2) (3) 肖良质。尔铁津 ${ }^{(4)}$

(1) 吉林大学物理系固体物理教研室, 长春 130023; (2) 中国科学院国际材料物理中心, 沈阳 110015; (3) 中国高等科学 技术中心 (世界实验室), 凝聚态和辐射物理分中心, 北京 100080; (4) 吉林大学化学系光化学教研室, 长春 130023)

\section{关键词 $\mathrm{FeOOH}$ 纳米固体 IR 光谱 合成压力 界面结构}

材料的性质是由材料的结构和内部的原子状态决定的. 纳米固体也不例外, 它的许多奇 异性质就是由其内部独特的界面结构决定的 ${ }^{[1 ~ 3]}$. 在纳米固体内部的界面上存在大量的不饱 合配位原子, 它们的键合形式多种多样, 形成了独特的界面结构. 由于压力作为纳米固体形 成的必要手段能有效地改变其内部的界面结构, 所以研究清楚压力对纳米固体结构的影响, 无论是对它的基础研究还是具体应用都是很重要的. 在本文中, 我们用 XRD 和 IR 谱研究了 成形压力对 $\mathrm{FeOOH}$ 纳米固体结构的影响.

以 $\mathrm{FeOOH}$ 超微粉 (TEM 形貌像显示粒径分布在 $3 \sim 24 \mathrm{~nm}$ 之间) 为原料, 经预压成形 后, 在大型两面顶环带式压机上分别用 3.0, 4.0, 4.8,6.0 GPa 的压力冷压合成纳米固体. 使 用日本 R.D/max-rA 大功率转靶 X 射线衍射仪测试纳米固体的 $X$ 射线衍射谱 (XRD); 使用 Nicolet. FTIR-5PC 型红外光谱仪测试纳米固体的远红外透射光谱 (IR).

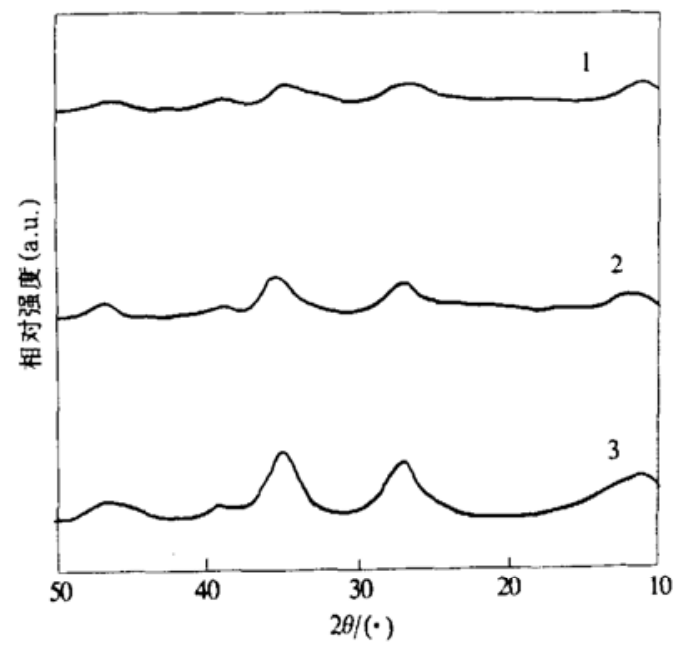

图 1 不同压力压制的纳米固体的室温 XRD 馏 $1-0.0 \mathrm{GPa}, 2-3.0 \mathrm{GPa}, 3-6.0 \mathrm{GPa}$

1994-12-19 收稿, 1995-04-27 收修改稿

*国家白然科学基金资助项日

图 1 给出了不同压力压制的纳米固体的室 温 XRD 谱. 在图 1-1所示的常压下超微粉的 XRD 谱中, 只在衍射角为 $11^{\circ}, 27^{\circ}, 35^{\circ}, 46^{\circ}$ 等处有强度较弱、宽度很宽的衍射漫峰, 其中心 位置与标准的 $\beta$-FeOOH 晶体衍射 (JCPDS 13157) 给出的衍射强峰位置相符, 表明超微粉的晶 体结构为 $\beta-\mathrm{FeOOH}$ 相. 将图 1-2, 3 与图 1-1 比 较可知, 随着压力增强, 衍射峰的峰位和峰宽度变 化不大, 但峰强明显增强. 这说明压力对晶粒内部 的唱体结构影响很小, 但对界面结构有明显的影 响, 使整体结构有序性增强.

图 2 给出了不同压力压制的纳米固体的室 温 IR 谱, 各谱主吸收峰的位置及相应的振动模 式 [4. 5]列于表 1. 图 2-1显示常压下超微粉的红 
表 1 不同压力压制的纳米固体的红外主吸收峰波数及相应的红外振动模式

\begin{tabular}{c|c|ccccccl}
\hline \multicolumn{2}{c|}{ 压力/GPa } & 0.0 & 3.0 & 4.0 & 4.8 & 6.0 & 块体 \\
\hline \multirow{3}{*}{ 波数/ } & I & 266.21 & 268.14 & 268.14 & 268.14 & 268.14 & $265 \sim 280$ & 振动模式 \\
$\mathrm{cm}^{-1}$ & II & 422.46 & 424.39 & 422.46 & 422.46 & 420.54 & $400 \sim 500$ & $\mathrm{~F}-\mathrm{O}-\mathrm{Oe}$ 对称 \\
& II & 677.10 & 679.03 & 680.96 & 682.89 & 682.89 & $640 \sim 700$ & $\mathrm{Fe}-\mathrm{O}-\mathrm{Fe}$ 非对称 \\
& IV & 846.86 & 844.93 & 846.86 & 848.79 & 850.72 & $840 \sim 860$ & $\mathrm{O}-\mathrm{H} \cdots \mathrm{O}$ 扭曲 \\
\hline
\end{tabular}

外吸收谱由四个主吸收峰 (I IV 以“O”表示) 和一些弱吸 收峰 (以“山”表示, 按强度由强到弱的顺序, 其对应波数分别为 $299,546,592,798,760,633$ 和 $390 \mathrm{~cm}^{-1}$ ) 组成. 表 1 和图 2 的 结果显示, 四个主吸收峰均对应 $\beta-\mathrm{FeOOH}$ 的晶格振动, 而且它 们的位置和相对强度随成形压力的增加都没有发生明显的变 化, 说明晶粒内部的晶体结构随压力变化不大, 这与上面 XRD 的结果是一致的. 但详细比较图 2-2, 3, 4 与图 1-1 可以看出, 加压以后那些位置处在 $\beta-\mathrm{FeOOH}$ 特征吸收区之外 (如表 1 所 示)的弱吸收峰发生了明显的变化: 随着压力升高, 它们的位置 基本保持不变, 但强度却逐渐减弱. 当压力达到 $6.0 \mathrm{GPa}$ 时, 这 些弱吸收峰都已基本消失, 纳米固体的红外光谱只剩下了属于 体相晶格振动的四个强吸收峰存在, 如图 2-4所示.

目前, 对纳米固体的界面结构主要有两种看法: 一种认为 是近于无序的 “类气态” 结构 ${ }^{[1,2]}$; 另一种认为界面结构仍具有短

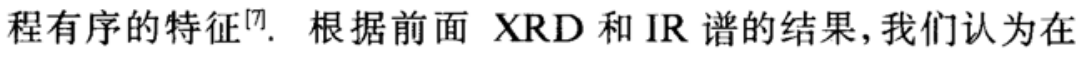
本文的样品中, 每个纳米颗粒都是由三部分结构组成的: 第一 部分是由近完整晶格组成的晶粒内核, 在其内部铁原子处于由 三个离子氧和三个羟基氧原子构成的轻微变形的八面体中心 ${ }^{[8]}$; 第二部分是由几乎无序的低配位的原子构成的表面原子层; 第 三部分是上述两部分结构之间的过渡区域, 在其中由于大量表 面原子的影响而使原子处于严重畸变的晶体配位环境中, 虽然

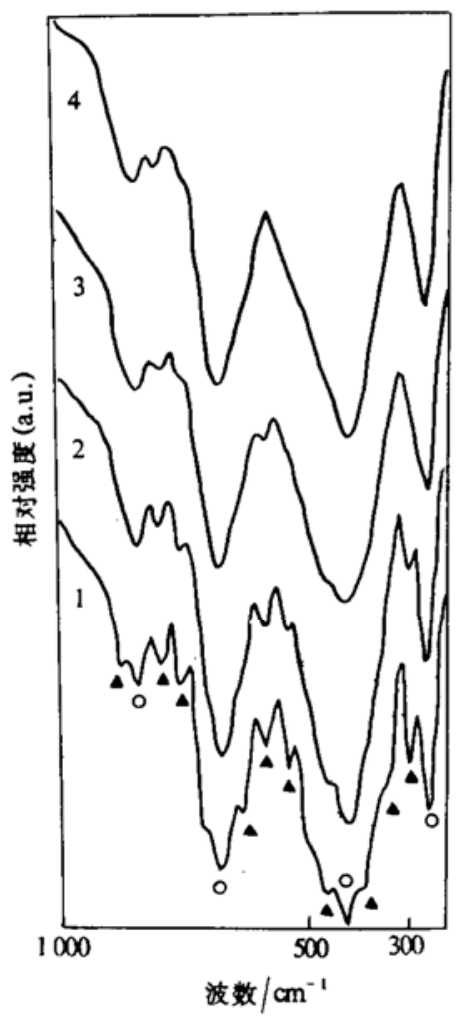

图 2 不同压力庄制的纳米固 体的室温 IR 谱

$1-0.0 \mathrm{GPa}, 2-3.0 \mathrm{GPa}, 3-$ $4.8 \mathrm{GPa}, 4-6.0 \mathrm{GPa}$ 这些原子的配位原子数目与完整晶格相近, 但它们的原子排列已失去原来的长程周期性而处 于某种短程有序状态.

基于上面的结构模型, 可以初步解释 $\beta-\mathrm{FeOOH}$ 纳米固体的 XRD 谱和 IR 谱随成形压力 的变化. 对于图 1-1所给出的超微粉的 XRD 谱, 晶体衍射峰主要由晶核内部有序排列的原 子的衍射给出, 而其它两部分原子的衍射没有规律, 不能形成衍射加强而淹没在谱中的背底 里. 对于超微粉的 IR 谱, 四个主吸收峰主要由晶核内部的原子振动给出; 另一方面, 在表面 低配位的原子形成了大量的悬键振动; 而在表面和晶核之间的过渡区域内, 严重的晶格渏变 使晶体对称性降低, 导致一些原来在高对称性下被禁止的红外振动模式重新显现出来. 与此 类似, 已有报道 ${ }^{[9]}$ 发现在 $\alpha-\mathrm{Al}_{2} \mathrm{O}_{3}$ 纳米粉体的红外吸收谱中存在二个强的禁止模式吸收, 并认 为它们是由晶粒内部严重的晶格变形所引起来的. 因此, $\beta-\mathrm{FeOOH}$ 超微粉的红外谱中异于 晶格振动吸收的弱卫星峰是由表面悬键振动和晶格畸变所致的禁止模式振动所引起的. 从 
图 2-1 可以看出, 这些卫星峰的数目很多且强度差异很大, 说明对于 $\mathrm{FeOOH}$ 这种具有大量羟 基集团的物质,在其超微粉表面存在大量弱的氢键, 它们的成键方式和强度多种多样, 从而导 致该超微粉的表面结构的无序程度可能要高于通常的纳米材料.

当超微粉被压制成纳米固体后, 它们的表面互相接触形成界面. 对于旵核内部的原子, 由于它们处在高对称性的饱合配位状态, 原子间的相互作用很强, 在我们所使用的压力范围 内, 压力还不足以使晶核内部的原子位置和间距发生明显的变化, 从而不能改变这部分唱体 结构和原子振动特征．另一方面, 界面上低配位原子在压力的作用下相互成键而趋于饱合配 位状态, 使悬键数日相应减少; 同时, 界面原子配位的增加使得表面和晶核间的过渡区内晶 格畸变减弱, 晶体对称性增强, 原子排列有序性增强. 这些变化导致随着压力升高, 纳米周体 的 XRD 谱中晶体衍射强度逐渐增强, 红外谱中对应悬键振动和禁止模式振动的吸收峰强度 逐渐减弱, 从而使得 $\beta$ - $\mathrm{FeOOH}$ 纳米固体在 $6.0 \mathrm{GPa}$ 压力下表现出接近正常多晶固体的结构 特征.

\section{参考文献}

I \pm 厂厚, 韩 民. 纳米微晶材料的结构和性质. 物理学进展, 1990, 10(3): 248 289

2 Gleiter H. Nanocrystalline materials prog. In Mat Sci, 1989, 33(4): 223 315

3 张立德, 牟季美. 开拓原子和物质的中间领域—— 纳米微粒与纳米固体. 物理, 1992, 21(3): 167 173

4 Gonzalez-Calbet J M, Alario-Franco M A, Gayoso-Andrade M. The porous structure of synthetic akaganeite. J Inorg Nucl Chem, 1981, 43: 257 264

5 Kauffman K, Hazel F. Infrared and Mössbauer spectroscopy, electron microscopy and chemical reactivity of ferric chloride hydrolysis products. J Inorg Nucl Chem, 1975, 37: 1139 1 148

6 Niquest R A. Infrared Spectra of Inorganic Compound. New York: Academic, 1971, 240 241

7 Zhang Lide, Mo Chimei, Wang Tao et al. Structure and bond properties of compacted and heat-treated silicon nitride particles. Phys Stat Sol (a), 1993, 136: 291 300

8 Das P, Bhattacharya D L. Study of environmental variation of $\mathrm{Fe}^{3+}$ Ions with temperature. J Mag Mag Mat, 1990 , 86: $102 \sim 104$

9 Mo C, Yuan Z, Zhang L et al. Infrared absorption spectra of nano-alumina. Nanostructured Materials, 1993, 2: $47 \sim 54$ 